
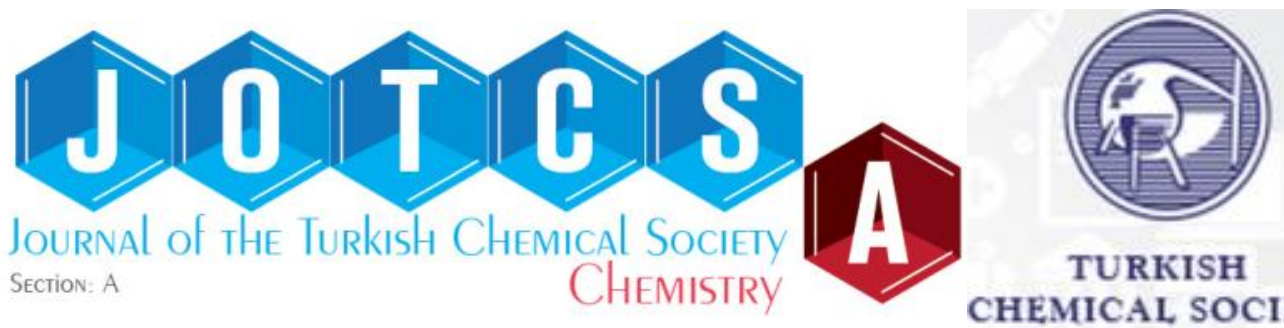

TURKISH

CHEMICAL SOCIETY

\title{
Preparation and Cytotoxicity of Coriandrum sativum L. Oil-Loaded Chitosan Nanoparticles
}

\section{Yasemin Budama-Kilinc ${ }^{1}$, Rabia Cakir-Koc ${ }^{1}$, Zeynep Kaya ${ }^{1}$}

1Yildiz Technical University, Chemical and Metallurgical Engineering Faculty, Bioengineering Department, 34220, Istanbul, Turkey.

\begin{abstract}
Coriandrum sativum L. (coriander) oil has antibacterial, antifungal, and antimicrobial activity. This study prepared and characterized chitosan nanoparticles loaded with the oil from Coriandrum sativum L., using an ionic gelation method with tripolyphosphate as the cross-linker. The coriander oil-loaded chitosan nanoparticles were characterized by Zeta Sizer measurements, Fourier transform infrared spectrometry, scanning electron microscopy (SEM), electron dispersive X-ray spectroscopy (EDS), and in vitro release properties. It was shown that chitosan nanoparticles loaded with coriander oil have an average size of $113.5 \mathrm{~nm}$, zeta potential of 16 $\mathrm{mV}$ and a polydispersity index of 0.378 . The encapsulation efficiency was calculated as $72 \%$. The spherical morphology of the nanoparticles was verified by SEM analysis, and EDS revealed the elemental composition. Furthermore, cytotoxicity analysis using L929 fibroblast cells and the XTT method demonstrated that coriander oil-loaded chitosan nanoparticles, coriander oil, and empty chitosan nanoparticles had no significant cell toxicity.
\end{abstract}

Keywords: Cell culture, chitosan, coriander (Coriandrum sativum L.), cytotoxicity, nanoparticle.

Submitted: July 20, 2017. Accepted: November 30, 2017.

Cite this: Budama-Kilinc Y, Cakir-Koc R, Kaya Z. Preparation and Cytotoxicity of Coriandrum sativum L. Oil-Loaded Chitosan Nanoparticles. JOTCSA. 2018;5(1):179-92.

DOI: http://dx.doi.org/10.18596/jotcsa.323798.

*Corresponding author. E-mail: yaseminbudama@gmail.com; Tel: +90 21238346 47; Fax: +902123834626. 


\section{INTRODUCTION}

Coriandrum sativum L. (coriander) is a member of the Apiaceae family and used as a medicinal plant (1). It is an annual, herbaceous plant grown for therapeutic purposes in North and Middle Africa and Asia (2). Coriander oil is one of the prominent essential oils found in the global market (3).

Various pharmacognostic, phytochemical, and pharmacological studies of coriander oil and its bioactive phenolic components have been published (1). Such literature investigations highlight the effectiveness of coriander oil as an antibacterial agent against gram-positive (Staphylococcus aureus, Bacillus spp.) and gram-negative bacteria (Escherichia coli, Salmonella typhi, Klebsiella pneumoniae, Proteus mirabilis, Pseudomonas aeruginosa) (4). In addition, its antimicrobial activity against pathogenic Candida albicans fungus has been illustrated, and it has been shown that coriander oil has significant antibacterial and antifungal activity against all microbes, except antibiotic-resistant $P$. aeruginosa (5).

The main component of coriander oil, linalool, has demonstrated to have anti-cancer activity (6). It is thought to increase the therapeutic index of anthracyclines in the therapy of breast cancer and especially in drug-resistant tumors (7). Other authors have reported that coriander nectar and volatile oils can display anti-inflammatory, as well as anti-oxidant properties (8). In vivo and in vitro assays indicate that the bioactive components of coriander have hypoglycemic, hypolipidemic, anxiolytic, analgesic, anti-inflammatory, anti-convulsant, anti-helmintic, and hepatoprotective activities (4). However, the use of herbal components is limited due to their low stability. Recently, development of nano-sized formulations, which encapsulate herbal constituents in polymer networks, has gained importance because it is known that the encapsulation techniques preserve the characteristics of medicinal herbal oils, as well as increase their activity due to their nano-size (9).

A wide range of polymers and various polymer combinations are suitable for the encapsulation of nanostructures. Chitosan is commonly used as a wall material polymer because it does not show toxicity. Further advantages of chitosan are its biocompatibility, due to its physical and chemical properties (10), biodegradability, and stability in micro- and nano-sized particles, and under high-temperature conditions (11). For these reasons, chitosan nanoparticles have great importance in controlled drug delivery systems $(12,13)$.

The antimicrobial activity of coriander oil microcapsules has been studied in a previous work (14). Another study determined the swelling and release attributes of coriander oil encapsulated in microparticles using various materials, such as chitosan, alginate, chitosan/alginate, and chitosan/insulin (15). 
However, encapsulation of coriander oil into nano-scale particles has not yet been documented. Therefore, this study aimed to prepare coriander oil-loaded chitosan nanoparticles and characterize their properties by using a Zeta Sizer, scanning electron microscope, Fourier transform infrared (FTIR) spectrometer, and an electron dispersive X-ray spectroscope. The in vitro cytotoxicity of the samples was also determined.

\section{MATERIALS AND METHODS}

\section{Chemicals}

Chitosan (75-85\% deacetylated, low molecular weight, CAS no. 9012-76-4), sodium tripolyphosphate (TPP, CAS no. 7758-29-4), and Tween 80 (CAS no. 9005-65-6) were purchased from Sigma-Aldrich (St. Louis, MO, USA). Acetic acid (CAS no. 64-19-7), coriander (Coriandrum sativum L.) oil (99\% pure), and 2,3-bis-(2-methoxy-4-nitro-5-sulfophenyl)-2H-tetrazolium-5carboxanilide (XTT) were obtained from Merck (Darmstadt, Germany). All the chemicals and solvents were of analytical grade and were used without further purification. Ultra-pure water was obtained using a Millipore Milli-Q Gradient System.

\section{METHODS}

\section{Preparation of coriander oil-loaded chitosan nanoparticles}

Coriander oil-loaded chitosan nanoparticles were prepared using a modified version of the ionic gelation method (16). Chitosan (2.5\% w:v) was added to $1 \%(\mathrm{v}: \mathrm{v})$ acetic acid solution and mixed on a magnetic stirrer until a homogeneous solution was obtained. The $\mathrm{pH}$ of the solution was adjusted to 4.6-4.8. Next, coriander oil and Tween 80 mixture ( $1: 1 \mathrm{v}: \mathrm{v}$ ) were combined with a solution of chitosan. In order to obtain a homogeneous solution, the mixture was stirred at room temperature for $2 \mathrm{~h}$. Next, TPP was dissolved in distilled water $(0.05 \%, \mathrm{w}: \mathrm{v})$ and then added drop-wise. The solution was agitated on a magnetic stirrer for $45 \mathrm{~min}$, and finally, ultrasonication was applied.

In order to prepare chitosan nanoparticles, the first two steps were performed as per the coriander oil-loaded nanoparticles. The remaining steps were performed with TPP, in equal amounts as described above, without coriander oil.

\section{Characterization studies}

Average size, polydispersity, and size distribution with dynamic light scattering (DLS): Photon correlation spectroscopy was accomplished using a Zeta Sizer Nano ZS (Malvern Instruments, UK) instrument, equipped with a $4.0 \mathrm{mV}$ He-Ne laser $(633 \mathrm{~nm})$, operating at $25^{\circ} \mathrm{C}$. This technique enabled assessment of the average size, polydispersity index (PDI), and size distribution of the coriander oil-loaded chitosan and chitosan nanoparticles. Before every 
electrophoretic light scattering measurement, each sample was filtered through a $0.2 \mu \mathrm{m}$ regenerated cellulose membrane (Sartorius, Germany) to remove any impurities from the solutions.

FTIR spectrometry: The IR Prestige 21 FTIR spectrometer (Shimadzu, Japan) was used for chemical analyses of the functional groups present in the samples. Measurements were carried out for coriander oil, chitosan nanoparticles, and coriander oil-loaded chitosan nanoparticles, in universal attenuated total reflectance (ATR) mode. The FTIR spectra were collected from eight scans per sample, over the range 4000 to $750 \mathrm{~cm}^{-1}$, at $4 \mathrm{~cm}^{-1}$ resolution.

Scanning electron microscopy (SEM) and electron dispersive $X$-ray spectroscopy (EDS): The morphology of the coriander oil-loaded chitosan nanoparticles was analyzed using a scanning electron microscope (Zeiss Supra $50 \mathrm{~V}$ ). Nanoparticle samples were fixed to metal stubs and sputtered with a gold-palladium mixture at a thickness of $100 \AA$. The imaging was conducted using an accelerating voltage of $20 \mathrm{kV}$.

Determination of encapsulation efficiency and loading efficiency: The standard calibration curve of coriander oil was established by using a UV-Vis spectrophotometer (Shimadzu UV-1280) at $273 \mathrm{~nm}$, to calculate the encapsulation efficiency. Freeze-dried coriander oil-loaded chitosan nanoparticles were mixed with $5 \mathrm{~mL}$ of $2 \mathrm{M}$ hydrochloric acid solution and boiled at $95^{\circ} \mathrm{C}$ for 30 min. After cooling, $2 \mathrm{~mL}$ of ethanol was added, and the solution then centrifuged at 9,000 rpm/25 ${ }^{\circ} \mathrm{C}$ for $5 \mathrm{~min}$ (17). The supernatant was analyzed by UV-Vis spectrophotometry at a wavelength of $273 \mathrm{~nm}$. The encapsulation efficiency of coriander oil-loaded chitosan nanoparticles was determined by calculating the concentration of the free coriander oil in the supernatant, from the coriander oil calibration curve.

$$
\text { Encapsulation Efficiency }(\%)=\frac{\text { Total drug amount-Free drug amount }}{\text { Total drug amount }} \times 100
$$

In vitro release experiment of coriander oil: To determine the in vitro coriander oil release profiles, the freeze-dried coriander oil-loaded chitosan nanoparticles $(20 \mathrm{mg}$ ) were placed in a tube containing a 3:2 ratio of PBS ( $\mathrm{pH} \mathrm{7.4)}$ and ethanol, and incubated at $37^{\circ} \mathrm{C}$ with gentle shaking. At specific time intervals, the samples were centrifuged at $9000 \mathrm{rpm} / 5^{\circ} \mathrm{C}$ for $5 \mathrm{~min}$ (18). The supernatant volume was taken for analysis, and the equivalent volume of fresh medium was added, followed by measurement at $273 \mathrm{~nm}$, using a UV-Vis spectrophotometer.

The release of coriander oil was determined as detailed in the following equation;

$$
\text { Release }(\%)=\frac{\text { Released coriander oil }}{\text { Total coriander oil }} \times 100
$$


Cytotoxicity experiments: An L929 cell line was used for the cytotoxicity studies. The mouse fibroblast cell line NCTC clone 929 (strain L) was derived in 1948 from a C3H/An male mouse. Fibroblast cultures are often used to evaluate materials intended for medical use. The cytotoxicity is taken as an indicator of bioincompatibility. Cells were cultured in a DMEM-F12 medium, supplemented with $10 \%$ fetal bovine serum and penicillin-streptomycin. The basal medium is widely used for supporting the growth of many different mammalian cells. The plates were incubated at $37{ }^{\circ} \mathrm{C}$ for 3 days in a $5 \% \mathrm{CO}_{2}$ incubator until confluency was attained. A trypsinization process was applied to the cells, and the detached cells were obtained by centrifugation.

The L929 cells were seeded (10,000 cells/well) in 96-well flat-bottom microplates with $100 \mu \mathrm{L}$ of medium. The plates were incubated at $37^{\circ} \mathrm{C}$ for $24 \mathrm{~h}$, for attachment to the well bottoms. Various concentrations $(3.12,6.25,12$, and $18 \mu \mathrm{g} / \mathrm{mL})$ of the chitosan nanoparticles, coriander, and coriander-oil-loaded chitosan nanoparticles were added, respectively, and incubated for another $24 \mathrm{~h}$. The media containing chitosan or nanoparticles were then aspirated, and $100 \mu \mathrm{L}$ of XTT solution in fresh medium was added to the wells at $0.5 \mathrm{mg} / \mathrm{mL}$ (with $7.5 \mu \mathrm{g} / \mathrm{mL}$ phenazine methosulfate). The plates were incubated at $37^{\circ} \mathrm{C}$ for $3 \mathrm{~h}$. Afterward, the optical density was measured at $450 \mathrm{~nm}$ with a multi-plate reader (Thermo Labsystems Multiscan Ascent 354 Microplate Photometer). Then, the percentage viability was calculated by the following equation:

$$
\text { Viability }(\%)=\frac{\text { Absorbance of experimental group }}{\text { Absorbance of control group }} \times 100
$$

\section{RESULTS AND DISCUSSION}

In this study, an ionic gelation technique was used to prepare the nanoparticles. Briefly, this method is based on an ionic gelation interaction, formed by the repulsive force between positively-charged chitosan and negatively-charged TPP. This procedure was undertaken to synthesize empty and coriander oil-loaded chitosan nanoparticles. These two types of nanoparticles were examined and compared for their physicochemical properties, such as particle size, zeta potential, and PDI, as well as their biological properties in cell culture.

The average size of the empty and coriander oil-loaded nanoparticles was determined using the DLS technique, with triplicate measurements (Table 1). As seen from Figures 1 and 2, the empty chitosan nanoparticles have a size of $156.2 \mathrm{~nm}$, a zeta potential of $22.4 \mathrm{mV}$, and a PDI of 0.150, whereas coriander oil-loaded chitosan nanoparticles are $113.5 \mathrm{~nm}$, with a zeta potential of 16 $\mathrm{mV}$ and a PDI of 0.378 . 
Budama-Kilinc, Cakir-Koc and Kaya, JOTCSA. 2018. 5(1); 179-190.

Table 1. Zeta Sizer results of nanoparticles

\begin{tabular}{cccc}
\hline Sample & Size $(\mathrm{nm})$ & PDI & $\begin{array}{c}\text { Zeta potential } \\
(\mathrm{mV})\end{array}$ \\
\hline Chitosan nanoparticles & $156.2 \pm 1.8$ & 0.150 & $22.4 \pm 1.95$ \\
$\begin{array}{c}\text { Coriander oil-loaded chitosan } \\
\text { nanoparticles }\end{array}$ & $113.5 \pm 3.5$ & 0.378 & $16.0 \pm 1.1$
\end{tabular}

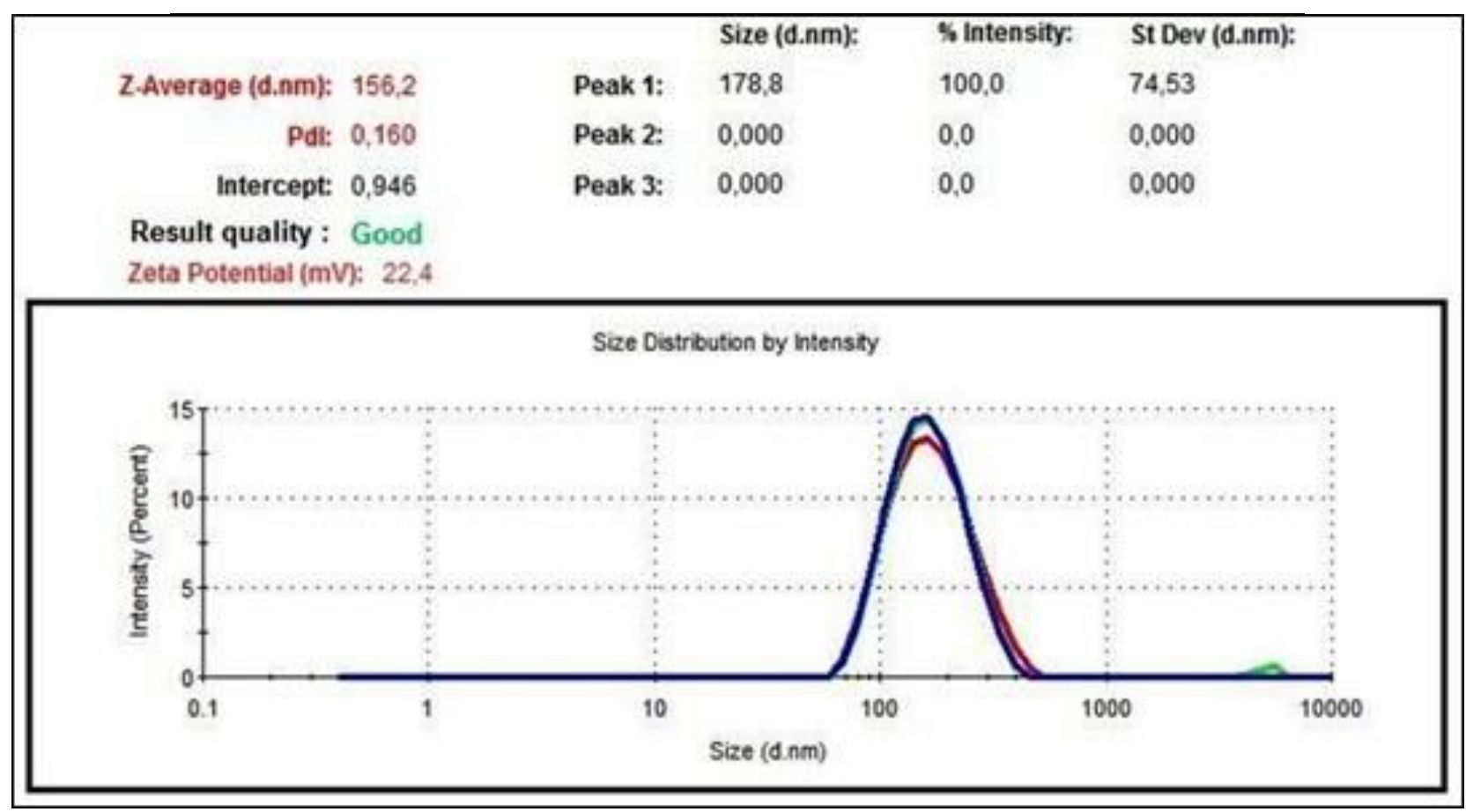

Figure 1. The Zeta Sizer result of chitosan nanoparticles.

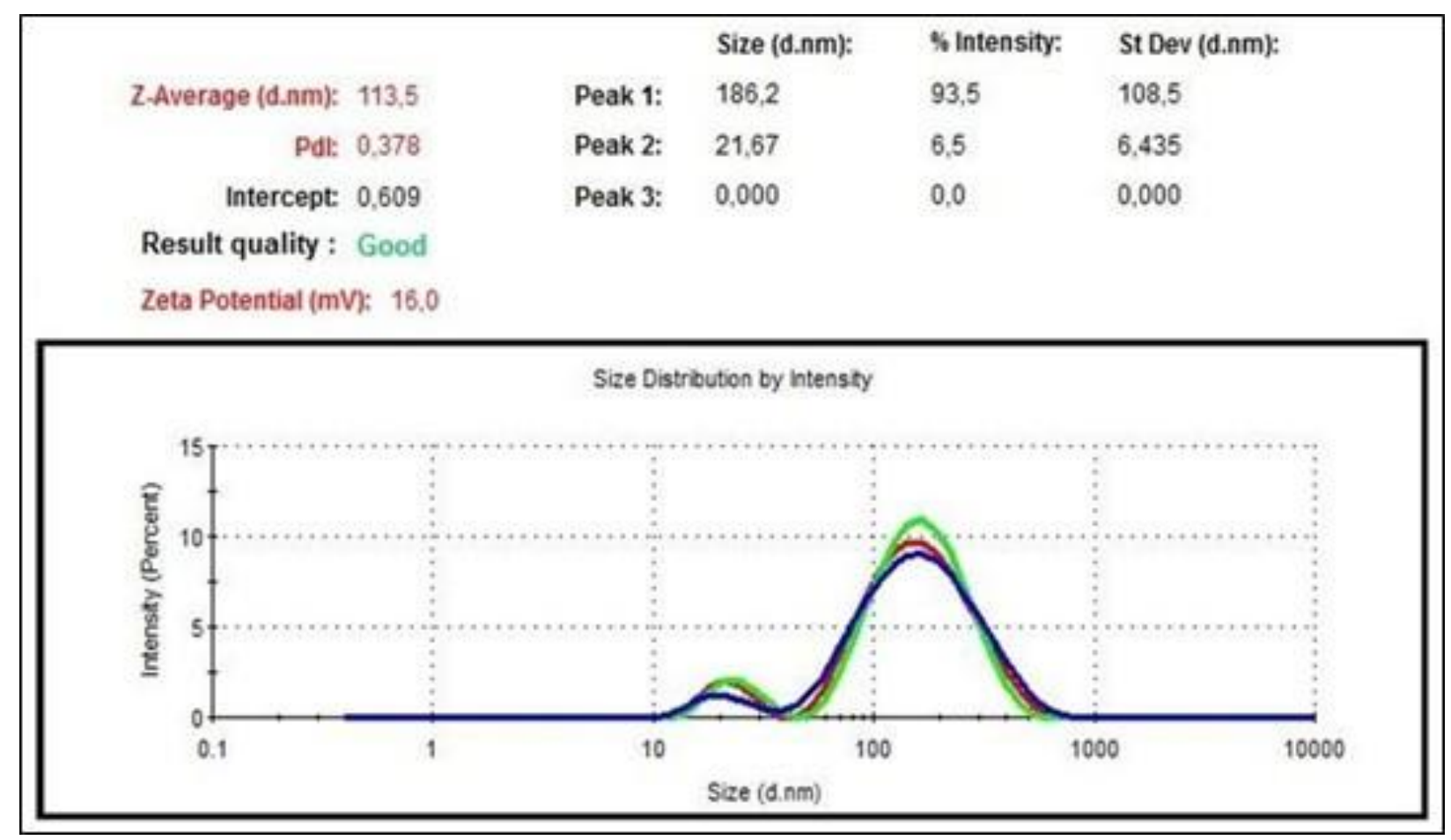

Figure 2. The Zeta Sizer result of coriander oil-loaded chitosan nanoparticles. 
During the encapsulation process, coriander oil and chitosan polymers are likely to interact with each other. Based on the weak interactions, such as hydrogen bonding, during this process, the conformation of the chitosan nanoparticles loaded with coriander oil may change. The DLS method showed that the size of the chitosan nanoparticles decreased when loaded with coriander. Thus, more compact structures have been formed with the loading of coriander oil into the chitosan nanoparticles.

The coriander oil-loaded chitosan nanoparticles were visualized, using a scanning electron microscope. As can be observed in Figure 3, coriander oil-loaded chitosan nanoparticles have a spherical morphology with some bridging flocculation between the particles.
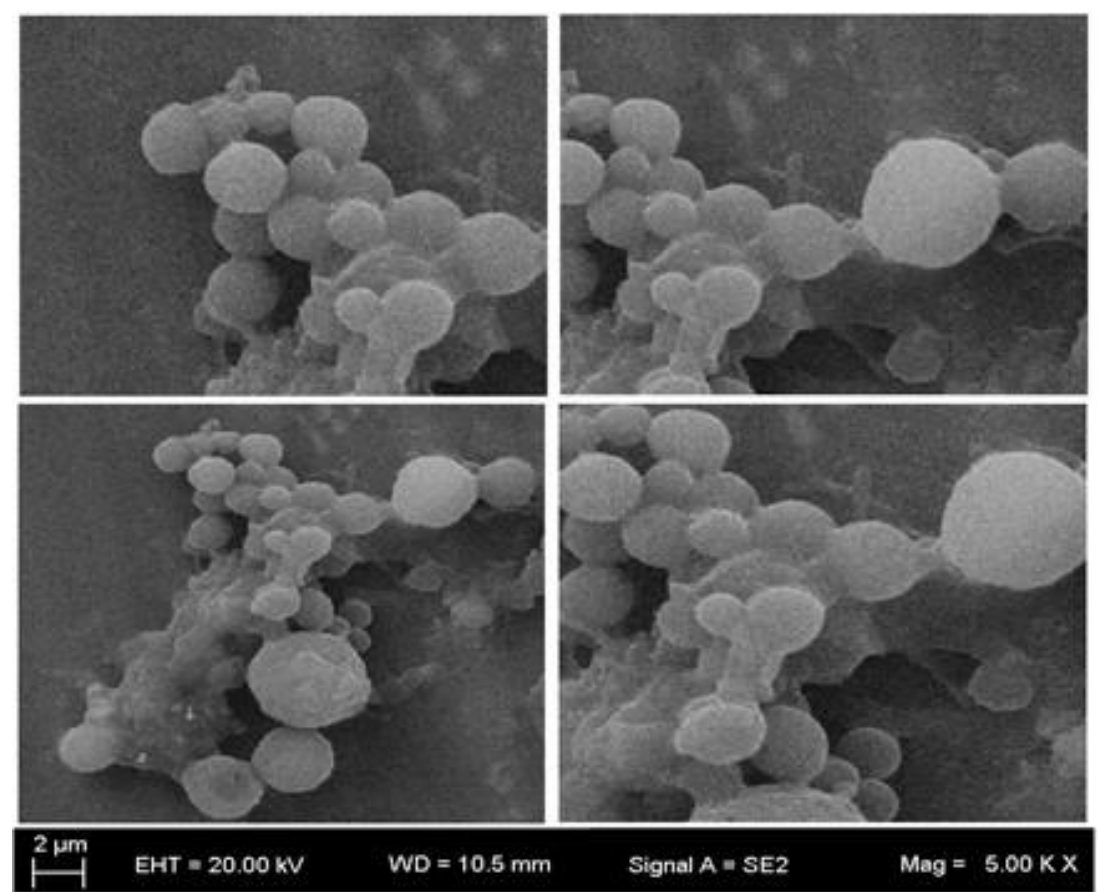

Figure 3. Scanning Electron Microscope images of coriander oil-loaded chitosan nanoparticles.

EDS is a chemical analysis method that can be used to determine the elemental composition at a certain point in the imaged area (19). Figure 4 provides the EDS results of the chitosan and coriander oil-loaded chitosan nanoparticles. The EDS spectra (Figure 4A) evidenced that the analysis site of the empty chitosan nanoparticles contained carbon (C), nitrogen ( $N$ ), and oxygen (O). All these elements are known to exist in the chitosan polymer structure. Similarly, for the two different points of the coriander oil-loaded chitosan nanoparticles analyzed by EDS, Figure $4 \mathrm{~B}$ reveals that $\mathrm{C}, \mathrm{N}$, and $\mathrm{O}$ are present. However, when compared to the empty chitosan nanoparticles, the intensities of these peaks were decreased, possibly due to the presence of coriander oil. 


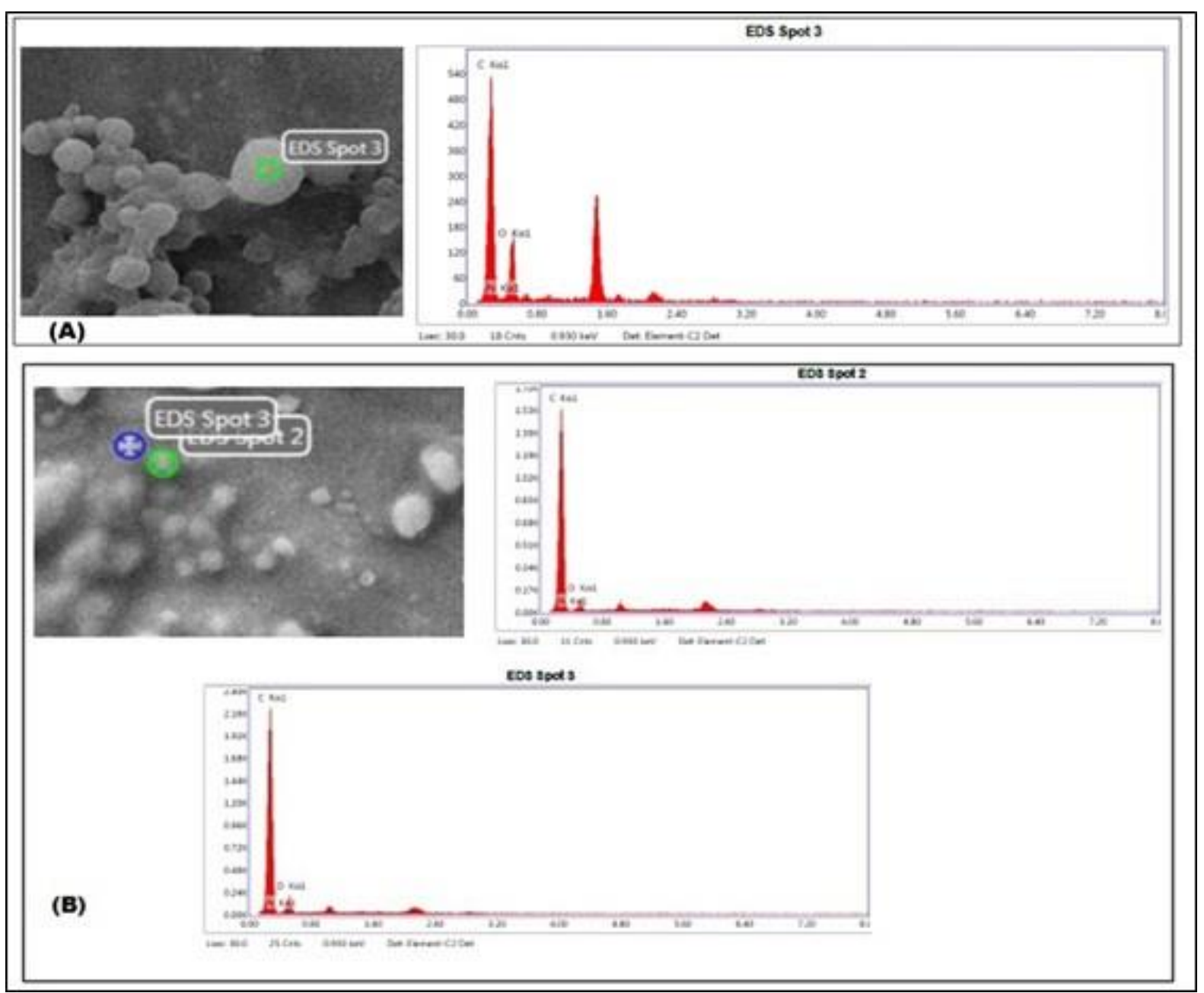

Figure 4. Scanning electron microscopy-electron dispersive X-ray spectroscopy results of chitosan (A) and coriander oil-loaded chitosan nanoparticles (B).

The nature of the chemical bonds present in the coriander oil, chitosan, and coriander oil-loaded chitosan nanoparticles were identified using FTIR. As illustrated in Figure 5, typically, the vibrations at $3005 \mathrm{~cm}^{-1}(-\mathrm{OH}), 2924 \mathrm{~cm}^{-1}(\mathrm{C}-\mathrm{H})$, and $2852 \mathrm{~cm}^{-1}(\mathrm{C}-\mathrm{H})$ observed in the coriander oil analysis are considered to originate from the hydrocarbon groups found in its chemical structure. There is a specific and intense peak at $1745 \mathrm{~cm}^{-1}$, arising from the ester groups $(C=O)$ (Figure 5(c)) and the signal at $1165 \mathrm{~cm}^{-1}$ is assigned to $\mathrm{C}-\mathrm{O}$ bonds. When the FTIR spectrum for chitosan nanoparticles was analyzed (Figure 5(a)), - NH vibrations at $1649 \mathrm{~cm}^{-1}$ and C-O signals at $1165 \mathrm{~cm}^{-1}$ were seen. In Figure 5(b), the FTIR results of the coriander oil-loaded chitosan nanoparticles indicate strong -OH vibrations at 3388 and $2922 \mathrm{~cm}^{-1}$. Compared to chitosan alone, coriander oil-loaded chitosan nanoparticles have a significantly sharper intensity at this position. At $1631 \mathrm{~cm}^{-1}$, an $-\mathrm{NH}$ peak characteristic of the amino group of chitosan is observed. A primary alcohol (C-O) peak specific to coriander oil at $1089 \mathrm{~cm}^{-1}$ and a $\mathrm{C}-\mathrm{H}$ peak at $871 \mathrm{~cm}^{-1}$ are also noticed. 


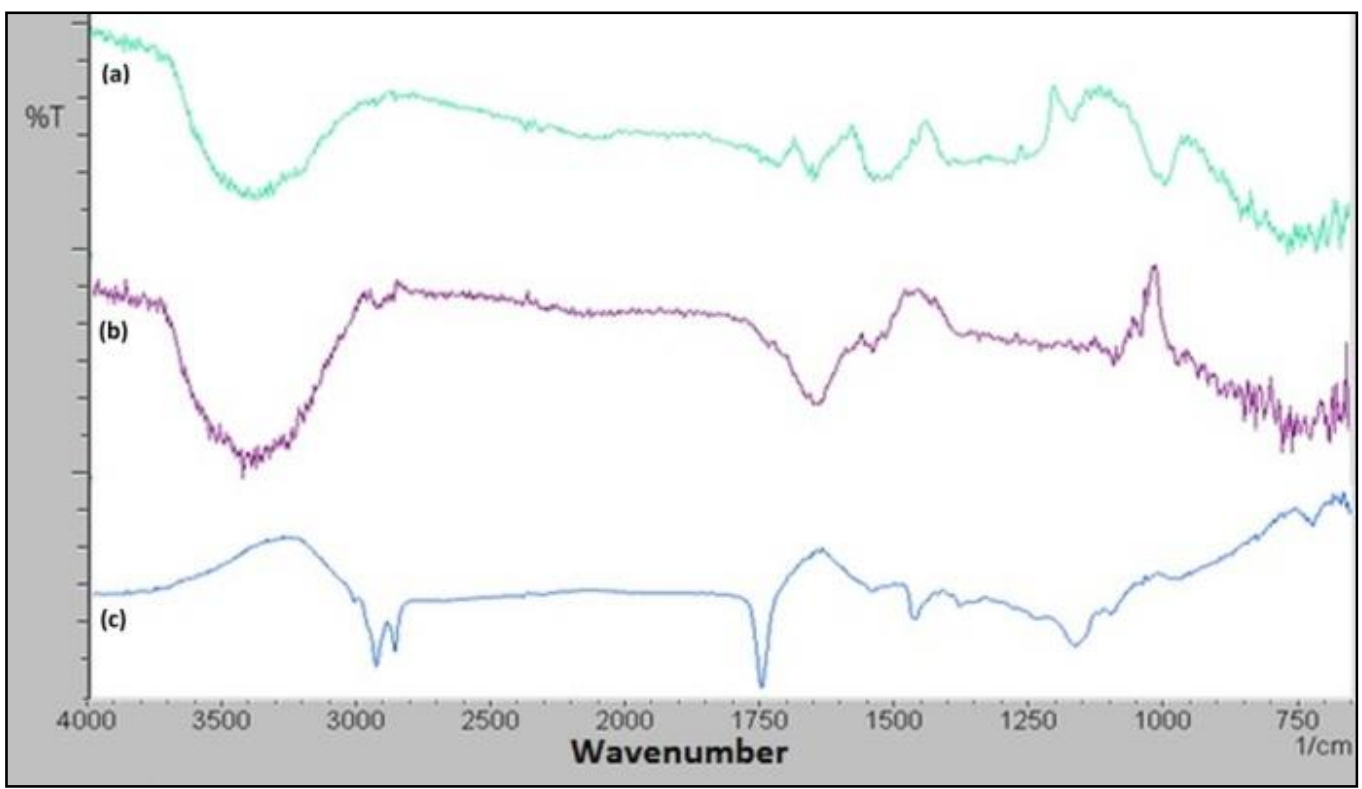

Figure 5. Fourier transform infrared spectra of chitosan nanoparticles (a), coriander oil-loaded chitosan nanoparticles (b), and coriander oil (c).

The standard graphic of coriander oil (Figure 6) was constructed, to calculate the encapsulation efficiency of the nanoparticles. The encapsulation of coriander oil into chitosan nanoparticles was confirmed by UV analysis at $273 \mathrm{~nm}$. The encapsulation efficiency of coriander oil was calculated as $72 \%$ (Eq. 1).

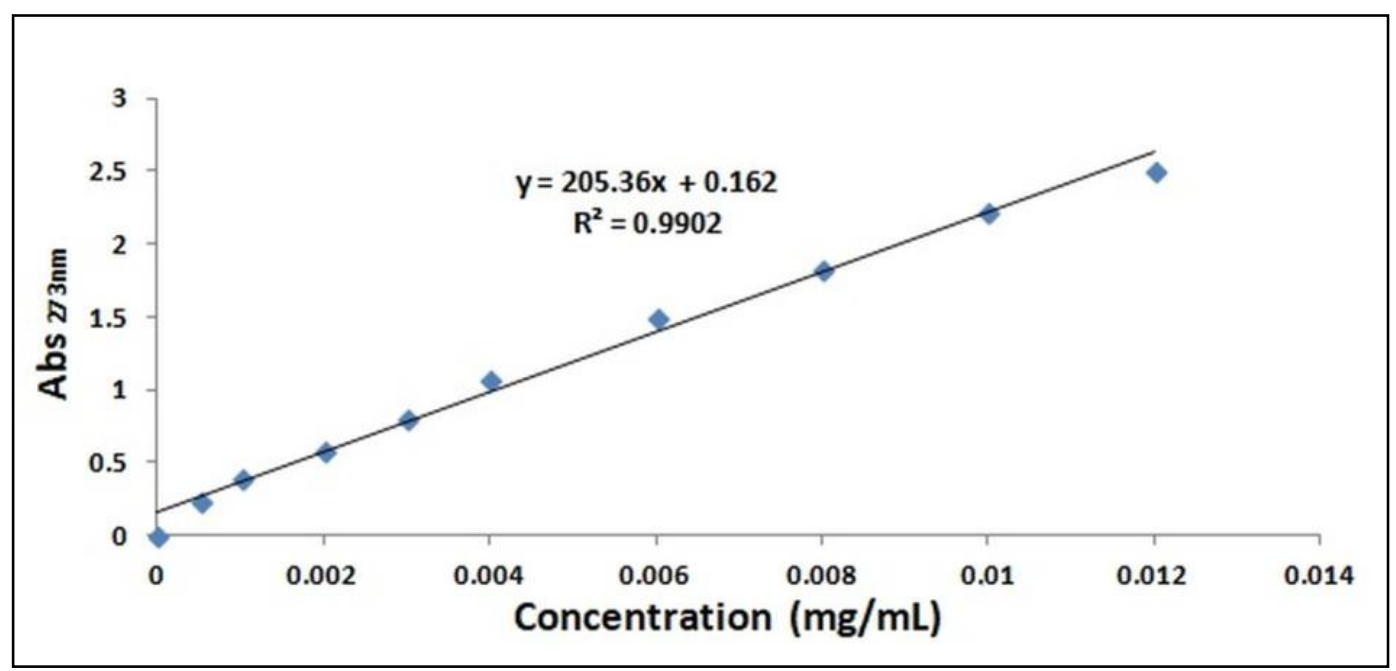

Figure 6. The standard calibration curve of coriander oil at $273 \mathrm{~nm}$.

In order to perform the in vitro release experiment, the free coriander oil concentration was calculated. The in vitro release profile of coriander oil from chitosan nanoparticles is depicted in Figure 7. The amount of coriander oil released at different times was measured by UV-Vis spectroscopy at $273 \mathrm{~nm}$. The experiment was performed for $5 \mathrm{~h}$, and $97 \%$ coriander oil was released at the end of the second hour. 


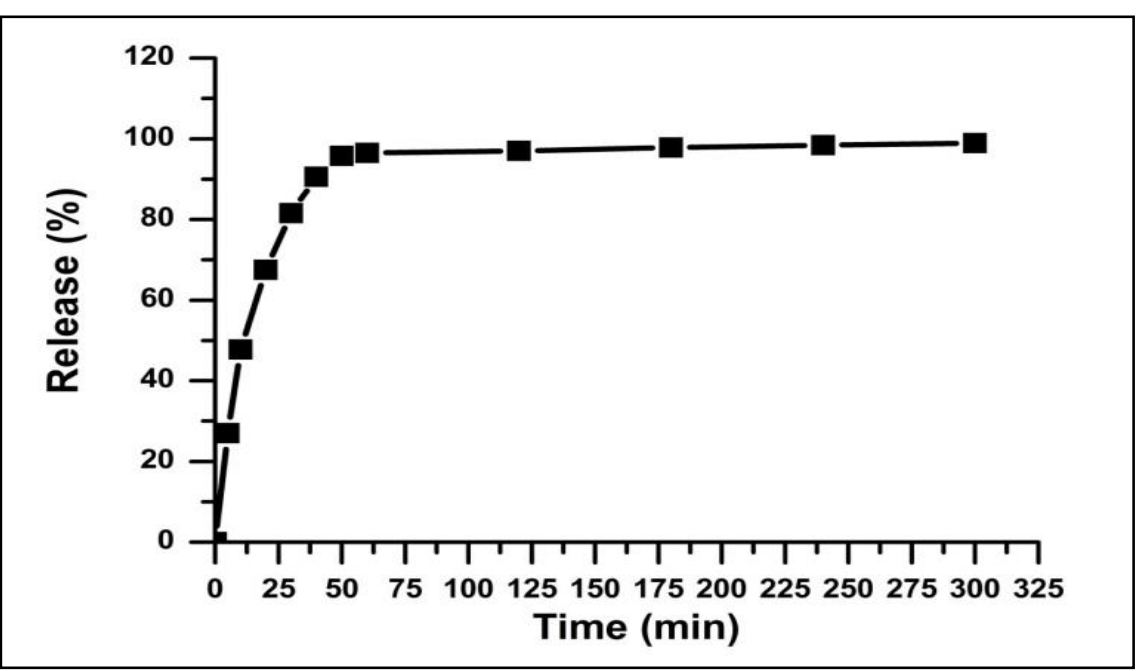

Figure 7. In vitro release profile of coriander oil-loaded chitosan nanoparticles.

After the characterization experiments of the nanoparticles, the coriander oil, coriander oilloaded chitosan nanoparticles, and chitosan nanoparticles were used, respectively, at four different concentrations $(3.12,6.25,12$, and $18 \mu \mathrm{g} / \mathrm{mL}$ ) in cell culture experiments (Figure 8). Coriander oil, indicated in Figure 8 by the green bars, did not provoke a significant toxic effect on the cells. Cell viability only decreased slightly at $18 \mu \mathrm{g} / \mathrm{mL}$ of coriander oil compared to the control (0 $\mathrm{mg} \mu \mathrm{g} / \mathrm{mL})$.

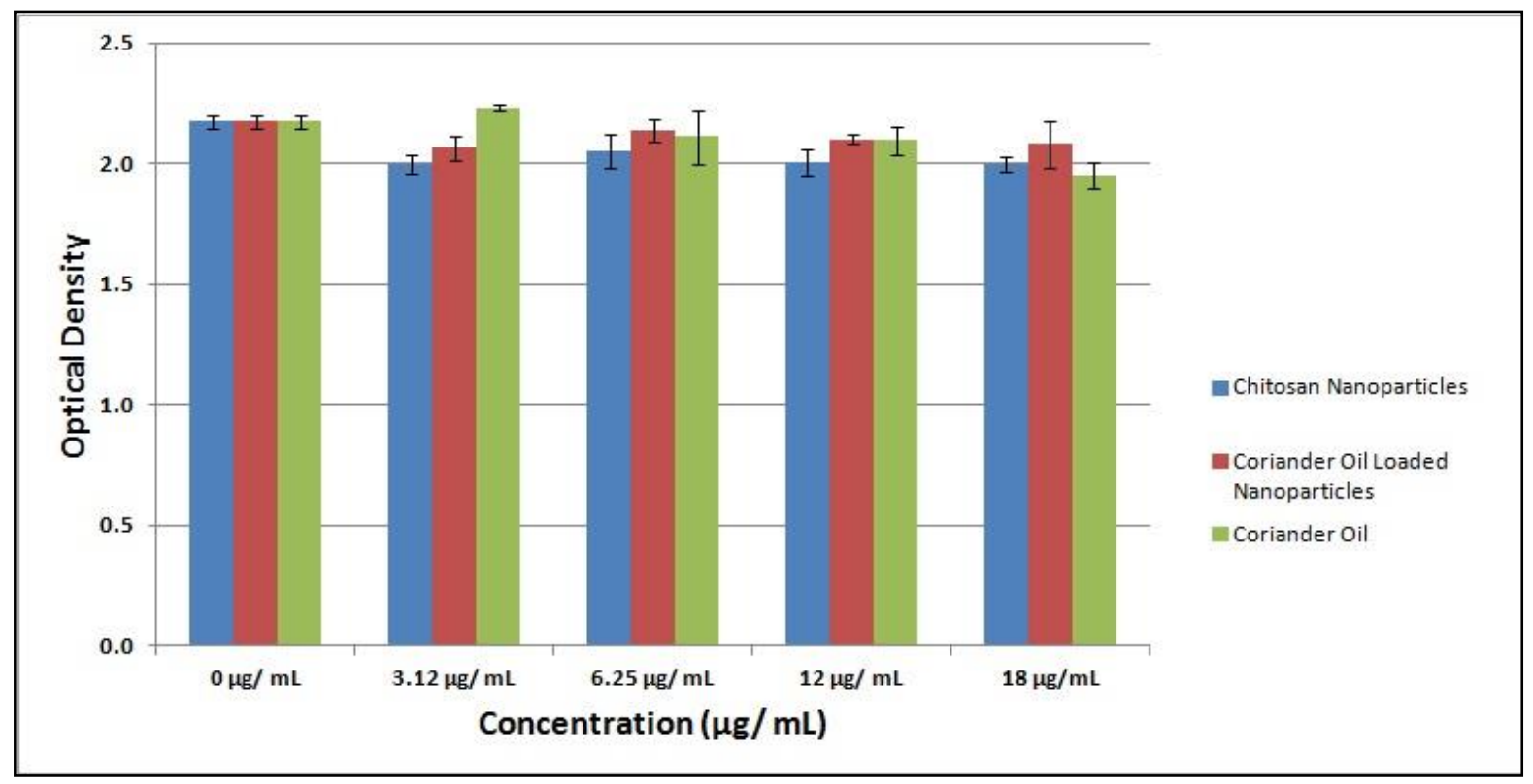

Figure 8. Differentiation of L929 cell viability with concentration.

Similarly, the coriander oil-loaded chitosan nanoparticles (Figure 8, red bars) presented no significant decrease in 2929 cell viability, at the concentrations investigated. It is known that chitosan is a biocompatible polymer and does not provoke a toxic effect on cells (20). Our cell culture results (Figure 8 , blue bars) also support this literature data. The cell viability was very similar to the control group. 
Table 2 shows the percentage effect on 2929 cell viability after $24 \mathrm{~h}$ treatment with coriander oil, chitosan, and coriander oil-loaded chitosan nanoparticles, compared to the control ( $0 \mu \mathrm{g} /$ $\mathrm{mL}$ ). It is noted that the cell viability is over $90 \%$, even at the longest exposure time investigated.

Table 2. Viability (\%) of L929 cells exposed to coriander oil, chitosan nanoparticles, and coriander oil-loaded chitosan nanoparticles at different concentrations.

\begin{tabular}{cccc}
\hline $\begin{array}{c}\text { Sample } \\
\text { concentration }\end{array}$ & $\begin{array}{c}\text { Coriander oil- } \\
\text { loaded chitosan } \\
\text { nanoparticles }\end{array}$ & $\begin{array}{c}\text { Chitosan } \\
\text { nanoparticles }\end{array}$ & $\begin{array}{c}\text { Coriander } \\
\text { oil }\end{array}$ \\
\hline $3.12 \mu \mathrm{g} / \mathrm{mL}$ & $96.47 \pm 2.41$ & $93.87 \pm 1.89$ & $126.14 \pm 18.60$ \\
$6.25 \mu \mathrm{g} / \mathrm{mL}$ & $100.47 \pm 1.92$ & $96.33 \pm 3.19$ & $99.13 \pm 5.27$ \\
$12 \mu \mathrm{g} / \mathrm{mL}$ & $98.65 \pm 0.88$ & $93.41 \pm 2.72$ & $98.50 \pm 2.77$ \\
$18 \mu \mathrm{g} / \mathrm{mL}$ & $97.66 \pm 4.00$ & $93.81 \pm 1.52$ & $91.60 \pm 2.66$ \\
\hline
\end{tabular}

\section{CONCLUSION}

In this study, coriander oil-loaded chitosan nanoparticles, which may be used for various purposes as active agents, were synthesized and characterized. Structural analysis was done by spectroscopy and imaging techniques, and nanoparticle formation was confirmed, with an encapsulation efficiency of $72 \%$. The in vitro release studies indicated that the coriander oil present in the chitosan nanoparticles was released at the end of the second hour. These results confirm that coriander oil-loaded chitosan nanoparticles were obtained successfully. Furthermore, cytotoxic effect of obtained coriander oil-loaded chitosan nanoparticles were investigated in comparison with coriander oil and empty chitosan nanoparticles on L929 cells in cell culture assays. According to ISO 10993-5 standards, percentages of cell viability above $80 \%$ are considered as non-cytotoxic; within $80 \%-60 \%$ weakly toxic; $60 \%-40 \%$ moderately toxic and below $40 \%$ strong cytotoxicity respectively $(21,22)$. Coriander oil-loaded chitosan nanoparticles were non-toxic, based on an in vitro assay with $L 929$ cells.

\section{CONFLICTS OF INTEREST}

The authors declare that they have no conflicts of interest.

\section{REFERENCES}

1. Laribi B, Kouki K, M'Hamdi M, Bettaieb T. Coriander (Coriandrum sativum L.) and its bioactive constituents. Fitoterapia. 2015;103:9-26. 
2. Diederichsen A. Coriander: Coriandrum Sativum L: Bioversity International; 1996.

3. Lawrence BM. Progress in essential oils. Perfumer \& flavorist. 1992;17(5):131-46.

4. Padmaa M. Coriandrum sativum linn: a review. Pharmacol online Newsl. 2009;3:561-73.

5. Matasyoh J, Maiyo Z, Ngure R, Chepkorir R. Chemical composition and antimicrobial activity of the essential oil of Coriandrum sativum. Food Chemistry. 2009;113(2):526-9.

6. Chang M-Y, Shen Y-L. Linalool exhibits cytotoxic effects by activating antitumor immunity. Molecules. 2014;19(5):6694-706.

7. Asgarpanah J, Kazemivash N. Phytochemistry, pharmacology and medicinal properties of Coriandrum sativum L. African Journal of Pharmacy and Pharmacology. 2012;6(31):2340-5.

8. Stashenko EE, Puertas MA, Martínez JR. SPME determination of volatile aldehydes for evaluation of invitro antioxidant activity. Analytical and bioanalytical chemistry. 2002;373(1):70-4.

9. Donsì F, Annunziata M, Sessa M, Ferrari G. Nanoencapsulation of essential oils to enhance their antimicrobial activity in foods. LWT-Food Science and Technology. 2011;44(9):1908-14.

10. Shukla SK, Mishra AK, Arotiba OA, Mamba BB. Chitosan-based nanomaterials: A state-of-the-art review. International journal of biological macromolecules. 2013;59:46-58.

11. Rinaudo M. Chitin and chitosan: properties and applications. Progress in polymer science. 2006;31(7):603-32.

12. Agnihotri SA, Mallikarjuna NN, Aminabhavi TM. Recent advances on chitosan-based micro-and nanoparticles in drug delivery. Journal of controlled release. $2004 ; 100(1): 5-28$.

13. Uhrich KE, Cannizzaro SM, Langer RS, Shakesheff KM. Polymeric systems for controlled drug release. Chemical reviews. 1999;99(11):3181-98.

14. Duman F, Kaya M. Crayfish chitosan for microencapsulation of coriander (Coriandrum sativum L.) essential oil. International Journal of Biological Macromolecules. 2016;92:125-33.

15. Dima C, Pătraşcu L, Cantaragiu A, Alexe P, Dima Ş. The kinetics of the swelling process and the release mechanisms of Coriandrum sativum L. essential oil from chitosan/alginate/inulin microcapsules. Food chemistry. 2016;195:39-48.

16. Calvo P, Remunan-Lopez C, Vila-Jato JL, Alonso M. Novel hydrophilic chitosan-polyethylene oxide nanoparticles as protein carriers. Journal of Applied Polymer Science. 1997;63(1):125-32.

17. Keawchaoon L, Yoksan, R. Preparation, characterization and in vitro release study of carvacrol-loaded chitosan nanoparticles,. Colloids and Surfaces B: Biointerfaces. 2011;84(1):163-71.

18. Hosseini S, Zandi, M., Rezaeia, M., Farahmandghavi, F. Two-step method for encapsulation of oregano essential oil in chitosan nanoparticles: Preparation, characterization and in vitro release study. Carbohydrate Polymers. 2013; 95(1):50-6.

19. Reed SJB, Reed SJB. Electron microprobe analysis: Cambridge University Press Cambridge; 1975.

20. Kumar MNR. A review of chitin and chitosan applications. Reactive and functional polymers. $2000 ; 46(1): 1-27$.

21. López-García J, Lehocký M, Humpolíček P, Sáha P. HaCaT keratinocytes response on antimicrobial atelocollagen substrates: extent of cytotoxicity, cell viability and proliferation. Journal of functional biomaterials. $2014 ; 5(2): 43-57$.

22. ISO. 10993-5:2009 Biological Evaluation of Medical Devices. Part 5: Tests for In Vitro Cytotoxicity. International Organization for Standardization. Geneva, Switzerland. 2009. 